\title{
Tools for Proving Zero Knowledge
}

\author{
Ingrid Biehl, Johannes Buchmann, \\ Bernd Meyer, Christian Thiel, Christoph Thiel \\ Universität des Saarlandes, Fachbereich Informatik, \\ Im Stadtwald 15, 6600 Saarbrücken, Germany
}

\begin{abstract}
We develop general techniques that can be used to prove the zero knowledge property of most of the known zero knowledge protocols. Those techniques consist in reducing the circuit indistinguishability of the output distributions of two probabilistic Turing machines to the indistinguishability of the output distributions of certain subroutines.
\end{abstract}

\section{Introduction}

It is an important result in the theory of zero knowledge proofs that assuming the existence of a circuit secure encryption machine every language in NP has a zero knowledge proof. This result can be obtained by constructing a zero knowledge proof system for the NP-complete language $3 \mathrm{C}$ of three colourable graphs (see $[1,2])$. In this protocol the prover and the verifier repeat a certain subprotocol a number of times which is polynomial in the length of the input. The encryption machine is called in a subroutine used in that subprotocol. The protocol can therefore be written in the form

$$
S=(M N O)^{n_{x}}
$$

where $M N O$ is the subprotocol which is repeated $n_{x}$ times, where $x$ is the input and $N$ is the subroutine which calls the encryption machine, and where $M$ and $O$ are the machines that carry out the computations before and after $N$ is used. In order to show that $S$ has the zero knowledge property one must show that the communication carried out in $S$ can be simulated by a probabilistic polynomial time Turing machine even if the verifier is replaced by a cheating verifier. After replacing the verifier the protocol is still of the form (1). The simulator $S^{\prime}$ is constructed by replacing $N$ with a machine $N^{\prime}$ which has no knowledge of a three colouring of the input graph. By virtue of the circuit security of the encryption machine, the output distributions of $N$ and $N^{\prime}$ are circuit indistinguishable. It remains to be shown that the output distributions of $S$ and $S^{\prime}$ are circuit indistinguishable.

Protocols and simulators for other zero knowledge protocols are constructed in the same way.

The goal of this paper is to unify the proofs for the zero knowledge property. We show that replacing the subroutine $N$ with $N^{\prime}$ in a probabilistic Turing machine $S$ of the form (1) yields (under certain conditions) a machine $S^{\prime}$ whose output distribution is circuit indistinguishable from the output distribution of 
$S$ if the output distribution of $N$ is circuit indistinguishable from the output distribution of $N^{\prime}$. Another goal of this paper is to precisely define the notions used in this context.

\section{Probabilistic Turing Machines}

Throughout this paper we use the alphabets $\Sigma=\{0,1, \#\}$ and $\Sigma_{0}=\{0,1\}$.

Definition 1. A probabilistic Turing machine is a pair $Z=(M, p)$ where

1. $M=(K, \Sigma, \Delta, s)$ is a $k$-tape nondeterministic Turing machine (see [3], pp. 204-211)

2. $p: \Delta \rightarrow[0,1]$ is a function which determines the probability of each transition in $\Delta$, i.e. for every $q \in K$ and $\mathbf{a} \in \Sigma^{k}$ we have

$$
\sum_{d \in \Delta(q, a)} p(d)=1
$$

where $\Delta(q, \mathbf{a})=\Delta \cap\left(\{q\} \times\{\mathbf{a}\} \times(K \cup\{h\}) \times(\Sigma \cup\{L, R\})^{k}\right)$.

A probabilistic Turing machine with $p(\Delta) \subseteq\left\{0, \frac{1}{2}, 1\right\}$ is called coin tossing machine.

We adopt the input and output conventions of [3]. For $x, y \in \Sigma_{0}^{*}$ we denote by $\Pi_{Z}(x, y)$ the probability for $Z$ to output $y$ on input of $x$. It is easy to see that

$$
\sum_{y \in \Sigma_{0}^{*}} \Pi_{Z}(x, y) \leq 1
$$

For $x \in \Sigma_{0}^{*}$ we denote by $Z(x)$ the set of all elements in $\Sigma_{0}^{*}$ that can with positive probability occur as an output of $Z$ on input of $x$, and $Z(L)=\bigcup_{x \in L} Z(x)$. If the length of each computation of $Z$ on input of $x$ is bounded by $c \in \mathbb{N}$, we denote the maximal length of a computation of $Z$ on input of $x$ by time $(Z(x))$. We say that the running time of $Z$ is bounded by a function $T: \mathbb{N} \rightarrow \mathbb{N}$ if for all $x \in \Sigma_{0}^{*}$ we have time $(Z(x)) \leq t(|x|)$.

If there is a function $\ell: \mathbb{N} \rightarrow \mathbb{N}$ such that, on input of strings of length $u$, the machine only outputs strings of length $\ell(u)$ with positive probability, then we call $Z$ a homogeneous probabilistic Turing machine.

If there is a polynomial $f \in \mathbb{N}[X]$ such that, on input of strings of length $u$, the output length of the machine $Z$ is bounded by $f(u)$, we call $Z$ a probabilistic Turing machine with polynomially bounded output length.

Let $Z_{1}$ and $Z_{2}$ be probabilistic Turing machines. Then the concatenation $Z_{1} Z_{2}$ of $Z_{1}$ and $Z_{2}$ is defined as the probabilistic Turing machine that first operates as $Z_{1}$. Whenever $Z_{1}$ terminates, $Z_{2}$ is called where the input of $Z_{2}$ is the output of $Z_{1}$. We also use the notation $Z_{1}^{n}$ for $\underbrace{Z_{1} Z_{1} \cdots Z_{1}}_{n \text { times }}$. 


\section{Probabilistic Circuits}

A probabilistic circuit is a deterministic circuit (see [4], pp. 73) with a partition In $=\operatorname{In}_{D} \cup \operatorname{In}_{P}, \operatorname{In}_{D} \cap \operatorname{In}_{P}=\varnothing$, of the input nodes. The input nodes in $\operatorname{In}_{D}$ (the deterministic input nodes) receive the input of the computation. The nodes in $\operatorname{In}_{P}$ (the probabilistic input nodes) are assigned uniformly at random 0 or 1 . The number of all nodes but the input nodes of a circuit $C$ is called size $(C)$.

For a probabilistic circuit $C$ with $m$ input nodes and $n$ output nodes and for $y \in\{0,1\}^{m}, z \in\{0,1\}^{n}$ we denote by $\Pi_{C}(y, z)$ the probability for $C$ to output $z$ on input of $y$.

Let $C_{1}$ be a probabilistic circuit with $n$ output nodes and $C_{2}$ be a probabilistic circuit with $n$ input nodes. Then the composition of $C_{1}$ and $C_{2}$ is the circuit which results by connecting the output nodes of $C_{1}$ with the input nodes of $C_{2}$.

Let $L \subseteq \Sigma_{0}^{*}$. A family $\left\{C_{x}\right\}_{x \in L}$ of probabilistic circuits is called polynomial if size $\left(C_{x}\right)$ is bounded by $|x|^{k}$ for some $k \in \mathbb{N}$.

In order to be able to prove our main theorems we need the following results.

Lemma 2. There is a constant $c \in \mathbb{N}$ such that for all Turing-decidable languages $L \subseteq\{0,1\}^{*}$ the following holds: if $L$ is decided by a deterministic Turing machine $M=(K, \Sigma, \delta, s)$ in time $T: \mathbb{N} \rightarrow \mathbb{N}$, then there is a family $\left\{C_{n}\right\}_{n \in \mathbb{N}}$ of deterministic circuits which decides $L$ and satisfies

$$
\operatorname{size}\left(C_{n}\right)=(|K||\Sigma|)^{e} T(n) \log T(n) .
$$

Proof. See [4], pp. 84-91.

Lemma 3. There are $c, d \in \mathbb{N}$ such that for all homogenous polynomial coin tossing machines $M=((K, \Sigma, \delta, s), p)$ with output length $\ell: \mathbb{N} \rightarrow \mathbb{N}$ and running time bounded by $T \in \mathbb{N}[X]$ there is a polynomial family $\left\{C_{n}\right\}_{n \in \mathbb{N}}$ of probabilistic ciresits such that $\left\{\Pi_{C_{|x|}}(x, \cdot)\right\}_{x \in L}$ and $\left\{\Pi_{M}(x, \cdot)\right\}_{x \in L}$ are equal and which satisfies

$$
\operatorname{size}\left(C_{n}\right)=d \ell(n)(|K||\Sigma|)^{c} T(n) \log T(n) .
$$

Proof. Without loss of generality we assume that there is $q \in \mathbb{N}[X]$ such that on input of length $n$ the machine $M$ tosses the coin exactly $q(n)$ times. Moreover, we can construct a homogeneous polynomial time deterministic Turing machine $M^{\prime}=\left(K^{\prime}, \Sigma, \delta^{\prime}, s^{\prime}\right)$ with the following property: suppose that on input of $x$ the machine $M$ carries out the sequence of coin tosses $\alpha=\left(\alpha_{1}, \ldots, \alpha_{q(|x|)}\right)$ and outputs $y$, then, one input of $(x, \alpha)$, the machine $M^{\prime}$ outputs $y$. There is a constant $r \in \mathbb{N}$ (independent of $M$ ) such that $\left|K^{\prime}\right| \leq r|K|$. There is an other constant $s \in \mathbb{N}$ (independent of $\left.M^{\prime}\right)$ such that $T^{\prime}(|(x, \alpha)|) \leq s T(|x|)$ for the running time $T^{\prime}$ of $M^{\prime}$.

If we define for $x, y \in \Sigma_{0}^{*}$

$$
\Pi_{M^{\prime}}(x, y)=\frac{1}{2^{q(|x|)}}\left|\left\{\alpha \in\{0,1\}^{g(|x|)} \mid M^{\prime}(x, \alpha)=y\right\}\right|
$$

then we have $\Pi_{M^{\prime}}=\Pi_{M}$. 
In order to be able to apply Lemma 2 we consider the deterministic Turing machine $M_{m}^{\prime}(m \in \mathbb{N})$ which on input of $(x, \alpha)$ outputs the $m$ th bit of the output $y$ which is defined to be 0 if $m>\ell(|x|)$.

For $x \in\{0,1\}^{*}, y=\left(y_{1}, \ldots, y_{\ell(|x|)}\right) \in\{0,1\}^{*}$ we have

$$
\Pi_{M^{\prime}}(x, y)=\frac{1}{2^{q(|x|)}}\left|\left\{\alpha \in\{0,1\}^{q(|x|)} \mid M_{i}^{\prime}(x, \alpha)=y_{i}, \forall 1 \leq i \leq \ell(|x|)\right\}\right| .
$$

The machine $M_{m}^{\prime}$ works exactly as $M^{\prime}$ and deletes at the end of its computation all but the $m$ th bit of the output. Therefore there is a constant $t \in \mathbb{N}$ (independent of $\left.M^{\prime}\right)$ such that $T_{m}^{\prime}(|(x, \alpha)|) \leq t T^{\prime}(|(x, \alpha)|)$ for the running time $T_{m}^{\prime}$ of $M_{m}^{\prime}$. The number of states of $M_{m}^{\prime}$ is polynomial in the number of states of $M$.

We apply Lemma 2 to $M_{m}^{\prime}$ and thus obtain a polynomial family $\left\{C_{n}^{(m)}\right\}_{n \in \mathbb{N}}$ of deterministic circuits which simulates $M_{m}^{\prime}$. The circuit $C_{n}^{(m)}$ has $n$ deterministic and $q(n)$ probabilistic input vertices.

We construct the circuit $C_{n}$ by connecting the $C_{n}^{(m)}, 1 \leq m \leq \ell(|x|)$, in the natural order in parallel, that means all circuits have the same deterministic and probabilistic input.

Since $C_{n}$ is constructed from $\ell(n)$ circuits whose size is polynomially bounded in $n$, the size of $C_{n}$ itself is bounded by a polynomial in $n$, which means that $\left\{C_{n}\right\}_{n \in \mathbb{N}}$ is a polynomial family of circuits. Moreover. we have by construction that for every $x \in\{0,1\}^{*}$

$$
\Pi_{C_{|x|}}(x, \cdot)=\Pi_{M}(x, \cdot)
$$

\section{Indistinguishability}

Let $U$ and $V$ be two probability distributions on $\Sigma_{0}^{*}$. The series

$$
\delta_{S}(U, V)=\sum_{y \in \Sigma_{0}^{*}}|U(y)-V(y)|
$$

is called the statistical difference between $U$ and $V$. In general it is impossible to determine in polynomial time that two probability distributions have a non zero statistical difference. Therefore one uses tools like probabilistic circuits and probabilistic algorithms (i.e. probabilistic Turing machines) to distinguish between probability distributions.

For a probabilistic circuit with $m$ input nodes and one output node we call

$$
\delta_{C}(U, V)=\left|\sum_{y \in \Sigma_{0}^{m}} \Pi_{C}(y, 1)(U(y)-V(y))\right|
$$

the circuit difference between $U$ and $V$ with respect to $C$. 
Finally, for a probabilistic Turing machine $Z$ we call the series

$$
\delta_{Z}(U, V)=\left|\sum_{y \in \Sigma_{0}^{*}} \Pi_{Z}(y, 1)(U(y)-V(y))\right|
$$

the algorithmical difference between $U$ and $V$ with respect to $Z$.

Definition 4. Let $L \subseteq \Sigma_{0}^{*}$, let $U=\left\{U_{x}\right\}_{x \in L}$ and $V=\left\{V_{x}\right\}_{x \in L}$ be two families of probability distributions on $\Sigma_{0}^{*}$.

1. The families $U$ and $V$ are called perfectly indistinguishable ( $p$-indistinguishable) if $U=V$.

2. The families $U$ and $V$ are called statistically indistinguishable (s-indistinguishable) if for every $k \in \mathbb{N}$

$$
\lim _{\substack{x \in L \\|x| \rightarrow \infty}}|x|^{k} \delta_{S}\left(U_{x}, V_{x}\right)=0
$$

3. The families $U$ and $V$ are called circuit indistinguishable (c-indistinguishable) if for every polynomial family $\left\{C_{x}\right\}_{x \in L}$ of probabilistic circuits $C_{x}$ and for every $k \in \mathbb{N}$ we have

$$
\lim _{\substack{x \in L \\|x| \rightarrow \infty}}|x|^{k} \delta_{C_{x}}\left(U_{x}, V_{x}\right)=0
$$

4. The families $U$ and $V$ are called algorithmically indistinguishable (a-indistinguishable) if for every polynomial time probabilistic Turing machine $Z$ and for every $k \in \mathbb{N}$ we have

$$
\lim _{\substack{x \in L \\|x| \rightarrow \infty}}|x|^{k} \delta_{Z}\left(U_{x}, V_{x}\right)=0
$$

Lemma 5. Let $L \subseteq \Sigma_{0}^{*}$. Let $Z$ and $Z^{\prime}$ be homogeneous probabilistic Turing machines. Assume that $Z$ has polynomial output length. If $\left\{\Pi_{Z}(x, \cdot)\right\}_{x \in L}$ and $\left\{\Pi_{Z^{\prime}}(x, \cdot)\right\}_{x \in L}$ are circuit indistinguishable then for all $x \in L$ but a finite set the elements of $Z(x)$ and $Z^{\prime}(x)$ are of the same length.

Proof. The case $|L|<\infty$ is trivial. So assume $|L|=\infty$. Assume that there is an infinite subset $L^{\prime} \subseteq L$ such that for every $x \in L^{\prime}$ the elements of $Z(x)$ and $Z^{\prime}(x)$ are of different length. For $x \in L$ let $C_{x}$ be the circuit with $m_{x}$ input nodes, $m_{x}$ being the length of the elements in $Z(x)$, which always outputs 1 . Then we have

$$
\delta_{C_{x}}\left(\Pi_{Z}(x, \cdot), \Pi_{Z^{\prime}}(x, \cdot)\right)=1
$$

for all $x \in L^{\prime}$, hence

$$
\lim _{\substack{|x| \rightarrow \infty \\ x \in L}}|x| \delta_{C_{x}}\left(U_{x}, V_{x}\right) \neq 0 .
$$




\section{The Main Theorems}

Let $L \subseteq \Sigma_{0}^{*}$. A family $\left\{Z_{x}=\left(\left(K_{x}, \Sigma_{x}, \delta_{x}, s_{x}\right), p_{x}\right)\right\}_{x \in L}$ of probabilistic Turing machines is called polynomial if there are $p, q, r \in \mathbb{N}[X]$ such that for all $x \in L$ and $y \in \Sigma_{0}^{*} \operatorname{time}\left(Z_{x}(y)\right) \leq p(|x|) q(|y|)$ and $\left|K_{x}\right|\left|\Sigma_{x}\right| \leq r(|x|)$.

Theorem 6. Let. $L \subseteq \Sigma_{0}^{*}$. Let $\left\{M_{x}\right\}_{x \in L}$ be a family of probabilistic Turing machines. Let $N$ and $N^{\prime}$ be homogeneous probabilistic Turing machines, $N^{\prime}$ having polynomial output length. We define $\Gamma=\bigcup_{x \in L} M_{x}(x)$. Let $\left\{O_{x}\right\}_{x \in L}$ be a polynomial family of homogenous coin tossing machines. Assume that the following conditions hold:

1. $|z| \geq|x|$ for all $x \in L$ and $z \in M_{x}(x)$.

2. For $x \in L$ all elements of $M_{x}(x)$ are of the same length.

3. $\left\{\Pi_{N}(u, \cdot)\right\}_{u \in \Gamma}$ and $\left\{\Pi_{N^{\prime}}(u, \cdot)\right\}_{u \in \Gamma}$ are c-indistinguishable.

Then $\left\{\Pi_{M_{x} N O_{x}}(x, \cdot)\right\}_{x \in L}$ and $\left\{\Pi_{M_{x} N^{\prime} O_{x}}(x, \cdot)\right\}_{x \in L}$ are $c$-indistinguishable.

Proof. For $x \in L$ we set $A_{x}=M_{x} N O_{x}$ and $B_{x}=M_{x} N^{\prime} O_{x}$. Let $x \in L$ and let $\left\{O_{x, n}\right\}_{n \in \mathbb{N}}$ be a polynomial family of circuits simularing the probabilistic Turing machine $O_{x}$ (see Lemma 3). Let $\bar{N}(u)=N(u) \cup N^{-}(u)$.

Let $I \in \mathbb{N}$ such that for every $u \in \Gamma,|u|>I$ the elements of $N(u)$ and $N^{\prime}(u)$ are of the same length. According to Lemma 5 such an $I$ exists. Let $x \in L$, $|x|>I$, the elements of $A_{x}(x)$ and $B_{x}(x)$ are of the same length, say $m_{x}$. To measure a non zero circuit difference between $\Pi_{A_{x}}(x, \cdot)$ and $\Pi_{B_{x}}(x, \cdot)$ a circuit $C$ must have $m_{x}$ input nodes. Let $\left\{C_{x}\right\}_{x \in L}$ be a polynomial family of circuits. We assume that $C_{x}$ has exactly $m_{x}$ input nodes.

For $u \in M_{x}(x)$ all elements in $\bar{N}(u)$ have the same length $\ell(u), \ell \in \mathbb{N}[X]$. Let $O_{x, u}^{\prime}=O_{x, \ell(u)} C_{x}$ be the composition of $O_{x, \ell(x)}$ and $C_{x}$. Now we have:

$$
\begin{aligned}
& \delta_{C_{x}}\left(\Pi_{A_{x}}(x, \cdot), \Pi_{B_{x}}(x, \cdot)\right) \\
& =\left|\sum_{y \in \Sigma_{0}^{m_{x}}} \Pi_{C_{x}}(y, 1)\left(\Pi_{M_{x} N O_{x}}(x, y)-\Pi_{M_{x} N^{\prime} O_{x}}(x, y)\right)\right| \\
& =\left|\sum_{y \in \Sigma_{0}^{m_{x}}} \Pi_{C_{x}}(y, 1) \sum_{u \in M_{x}(x)} \sum_{v \in \bar{N}(u)} \Pi_{M_{x}}(x, u)\left(\Pi_{N}(u, v)-\Pi_{N^{\prime}}(u, v)\right) \Pi_{O_{x}}(v, y)\right| \\
& =\left|\sum_{u \in M_{x}(x)} \Pi_{M_{x}}(x, u) \sum_{v \in \bar{N}(u)} \sum_{y \in \Sigma_{0}^{m_{x}}} \Pi_{O_{x}}(v, y) \Pi_{C_{x}}(y, 1)\left(\Pi_{N}(u, v)-\Pi_{N^{\prime}}(u, v)\right)\right| \\
& =\left|\sum_{u \in M_{x}(x)} \Pi_{M_{x}}(x, u) \sum_{v \in \bar{N}(u)} \Pi_{O_{x, v}^{\prime}}(v, 1)\left(\Pi_{N}(u, v)-\Pi_{N^{\prime}}(u, v)\right)\right| \\
& \leq \sum_{u \in M_{x}(x)} \Pi_{M_{x}}(x, u) \delta_{O_{x}^{\prime}, v}\left(\Pi_{N}(u, \cdot), \Pi_{N^{\prime}}(u, \cdot)\right) .
\end{aligned}
$$


Let $u \in \Sigma_{0}^{*}$ and let $x^{\prime} \in L$ such that $u \in M_{x^{\prime}}\left(x^{\prime}\right)$ then $\left|x^{\prime}\right| \leq|u|$. Hence there are only finitely many $x^{\prime} \in L$ with $u \in M_{x^{\prime}}\left(x^{\prime}\right)$. Among the finitely many circuits $O_{x^{\prime}, u}^{\prime}, u \in M_{x^{\prime}}\left(x^{\prime}\right)$, we denote by $O_{u}^{\prime}$ the circuit which maximizes $\delta_{\alpha^{\prime}, u}^{\prime}\left(\Pi_{N}(u, \cdot), \Pi_{N^{\prime}}(u, \cdot)\right)$. Then we have

$$
\delta_{C_{x}}\left(\Pi_{A_{x}}(x, \cdot), \Pi_{B_{x}}(x, \cdot)\right) \leq \max _{u \in M_{x}(x)} \delta_{O_{u}^{\prime}}\left(\Pi_{N}(u, \cdot), \Pi_{N^{\prime}}(u, \cdot)\right)
$$

Now we consider $\operatorname{size}\left(O_{u}^{\prime}\right)$ :

$$
\begin{aligned}
\operatorname{size}\left(O_{u}^{\prime}\right) & \leq \max _{\substack{x \in E \\
|x \in<| u \mid}} \operatorname{size}\left(O_{x, u}^{\prime}\right)=\max _{\substack{x \in L \\
|x| \leq|x|}} \operatorname{size}\left(O_{x, \ell(u)} C_{x}\right) \\
& \leq \max _{\substack{x \in E \\
|x| \leq|u|}}\left(\operatorname{size}\left(O_{x, \ell(u)}\right)+\operatorname{size}\left(C_{x}\right)\right) .
\end{aligned}
$$

Since $\left\{O_{x}\right\}_{x \in L}$ is a polynomial family of coin tossing machines and $|x| \leq|u|$ there is (according to Lemma 3 ) a $p \in \mathbb{N}[X]$ such that for all $u \in M_{x}(x)$ $\operatorname{size}\left(O_{u}^{\prime}\right) \leq p(|u|)$. This implies with (2) that

$$
\begin{aligned}
& \lim _{\substack{x \in L \\
|x| \rightarrow \infty}}|x|^{k} \delta_{C_{x}}\left(\Pi_{A_{x}}(x, \cdot), \Pi_{B_{x}}(x, \cdot)\right) \\
& \leq \lim _{\substack{x \in L \\
|x| \rightarrow \infty}} \max _{u \in M_{x}(x)}|u|^{k} \delta_{O_{u}^{\prime}}\left(\Pi_{N}(u, \cdot), \Pi_{N^{\prime}}(u, \cdot)\right) \\
& =0 .
\end{aligned}
$$

Let $L \subseteq \Sigma_{0}^{*}$ be a language and let $\left\{n_{x}\right\}_{x \in L}$ be a sequence in $\mathbb{N}$. That sequence is called polynomially bounded if there is $d>0$ such that for every $x \in L$ we have $n_{x} \leq|x|^{d}$.

Theorem 7. Let $L \subseteq \Sigma_{0}^{*}$. Let $\left\{n_{x}\right\}_{x \in L}$ be a polynomially bounded sequence in NN. Let $S$ and $T$ be homogeneous probabilistic Turing machines. Assume that the following conditions hold:

1. $|y| \geq|x|$ for all $x \in L$ and $y \in S(x)$.

2. $S(L) \subseteq L$.

3. $T$ is a coin tossing machine such that for $q \in \mathbb{N}[X]$ and for all $i \in \mathbb{N}$ we have time $\left(T^{i}(z)\right) \leq i q(|z|)$.

4. $\left\{I_{S}(x, \cdot)\right\}_{x \in L}$ and $\left\{\Pi_{T}(x, \cdot)\right\}_{x \in L}$ are c-indistinguishable.

Then $\left\{H_{S^{n_{x}}}(x, \cdot)\right\}_{x \in L}$ and $\left\{\Pi_{T_{n_{x}}}(x, \cdot)\right\}_{x \in L}$ are c-indistinguishable.

Proof. We have

$$
\delta_{C_{x}}\left(\Pi_{S^{n_{x}}}(x, \cdot), \Pi_{T^{n_{x}}}(x, \cdot)\right)=\left|\sum_{y \in \Sigma_{0}^{m_{x}}} \Pi_{C_{x}}(y, 1)\left(\Pi_{S^{n_{x}}}(x, y)-\Pi_{T^{n_{x}}}(x, y)\right)\right| .
$$


We can write

$$
\Pi_{S^{n_{x}}}(x, y)-\Pi_{T^{n_{x}}}(x, y)=\sum_{i=0}^{n_{x}-1} \Pi_{S^{n_{x}-1} T_{i}^{i}}(x, y)-\Pi_{S^{n_{x}-i-1} T^{i+1}}(x, y)
$$

and therefore

$$
\begin{aligned}
& \delta_{C_{x}}\left(\Pi_{S^{n_{x}}}(x, \cdot), \Pi_{T^{n_{x}}}(x, \cdot)\right) \\
& =\left|\sum_{y \in \Sigma_{0}^{m_{x}}} \Pi_{C_{x}}(y, 1)\left(\sum_{i=0}^{n_{x}-1} \Pi_{S^{n_{x}-i} T^{i}}(x, y)-\Pi_{S^{n_{x}-i-1} T^{i+1}}(x, y)\right)\right| \\
& =\left|\sum_{i=0}^{n_{x}-1} \sum_{y \in \Sigma_{0}^{m_{x}}} \Pi_{C_{x}}(y, 1)\left(\Pi_{S^{n_{x}-i} T^{i}}(x, y)-\Pi_{S^{n_{x}-i-1} T^{i+1}}(x, y)\right)\right| \\
& \leq \sum_{i=0}^{n_{x}-1}\left|\sum_{y \in \Sigma_{0}^{m_{x}}} \Pi_{C_{x}}(y, 1)\left(\Pi_{S^{n_{x}-i} T^{i}}(x, y)-\Pi_{S^{n_{x}-i-1} T^{i+1}}(x, y)\right)\right| \\
& \leq n_{x} \max _{0 \leq i \leq n_{x}-1} \delta_{C_{x}}\left(\Pi_{S^{n_{x}-i-1} S T^{i}}(x, \cdot), \Pi_{S^{x_{x}-1-1} T_{T^{i}}}(x, \cdot)\right),
\end{aligned}
$$

i.e. for $x \in L$ there is $0 \leq i_{x} \leq n_{x}-1$ with

$$
\begin{aligned}
\delta_{C_{x}}\left(\Pi_{S^{n_{x}}}\right. & \left.(x, \cdot), \Pi_{T^{n_{x}}}(x, \cdot)\right) \\
& \leq n_{x} \delta_{C_{x}}\left(\Pi_{S^{n_{x}-i_{x}-1} S T^{x_{x}}}(x, \cdot), \Pi_{S^{n_{x}-i_{x}-1} \operatorname{TT}^{x_{x}}}(x, \cdot)\right) .
\end{aligned}
$$

Let $M_{x}=S^{n_{x}-i_{x}-1}, N=S, N^{\prime}=T$ and $O_{x}=T^{i x}$. We know:

1. $M_{x}$ being a concatenation of homogeneous Turing machines is also homogeneous. Since we have $|y| \geq|x|$ for $y \in S(x)$, we find $|y| \geq|x|$ for all $y \in M_{x}(x)$.

2. $N^{\prime}$ has polynomial output length.

3. The families $\left\{\Pi_{N}(x, \cdot)\right\}_{x \in L}$ and $\left\{\Pi_{N^{\prime}}(x, \cdot)\right\}_{x \in L}$ are $c$-indistinguishable. The set $\Gamma=\bigcup_{x \in L} M_{x}(x)$ is a subset of $L$ and therefore $\left\{\Pi_{N}(x, \cdot)\right\}_{x \in \Gamma}$ and $\left\{\Pi_{N^{\prime}}(x, \cdot)\right\}_{x \in \Gamma}$ are $c$-indistinguishable, too.

4. $O_{x}$ has the same alphabet as $T$. The set of states of $O_{x}$ is at most $n_{x}$-times the size of the set of states of $T$. Being a concatenation of homogeneous Turing machines, $O_{x}$ itself is a homogeneous Turing machine. Therefore $\left\{O_{x}\right\}_{x \in L}$ is a polynomial family of homogeneous coin tossing machines.

Therefore we can apply Theorem 6 . Using (3) we have for every $k \in \mathbb{N}$

$$
\begin{aligned}
& \lim _{\substack{|x| \rightarrow \infty \\
x \in L}}|x|^{k} \delta_{C_{x}}\left(\Pi_{S^{n_{x}}}(x, \cdot), \Pi_{T^{n_{x}}}(x, \cdot)\right) \\
& \leq \lim _{\substack{|x| \rightarrow \infty \\
x \in L}} n_{x}|x|^{k} \delta_{C_{x}}\left(\Pi_{M_{x} N O_{x}}(x, \cdot), \Pi_{M_{x} N^{\prime} O_{x}}(x, \cdot)\right) \\
& \quad\left(\left\{n_{x}\right\}_{x \in L} \text { is polynomially bounded. Therefore there is } d \in \mathbb{N} \text { with } n_{x} \leq|x|^{d}\right) \\
& \leq \lim _{\substack{|x| \rightarrow \infty \\
x \in L}}|x|^{d+k} \delta_{C_{x}}\left(\Pi_{M_{x} N O_{x}}(x, \cdot), \Pi_{M_{x} N^{\prime} O_{x}}(x, \cdot)\right) \\
& =0 .
\end{aligned}
$$


Thus $\left\{\Pi_{S^{n_{x}}}(x, \cdot)\right\}_{x \in L}$ and $\left\{\Pi_{T^{n_{x}}}(x, \cdot)\right\}_{x \in L}$ are $c$-indistinguishable.

Theorem 8. Let $L \subseteq \Sigma_{0}^{*}$. Let $\left\{n_{x}\right\}_{x \in L}$ be a sequence in $\mathbb{N}$. Let $S$ and $T$ be coin tossing machines. Assume that $S(L) \subseteq L$. If $\left\{\Pi_{S}(x, \cdot)\right\}_{x \in L}$ and $\left\{\Pi_{T}(x, \cdot)\right\}_{x \in L}$ are $p$-indistinguishable, then also $\left\{\Pi_{S^{n_{x}}}(x, \cdot)\right\}_{x \in L}$ and $\left\{\Pi_{T^{n_{x}}}(x, \cdot)\right\}_{x \in L}$.

Proof.

$$
\begin{aligned}
& \Pi_{S^{n_{x}}}(x, y)-\Pi_{T^{n_{x}}}(x, y) \\
& =\sum_{i=0}^{n_{x}-1} \Pi_{S^{n_{x}-i} T^{i}}(x, y)-\Pi_{S^{n_{x}-i-1} T^{i+1}}(x, y) \\
& =\sum_{i=0}^{n_{x}-1} \sum_{u \in \Sigma_{0}^{*}} \sum_{v \in \Sigma_{0}^{*}} \Pi_{S^{n_{x}-i-1}}(x, u) \underbrace{\left(\Pi_{S}(u, v)-\Pi_{T}(u, v)\right)}_{=0} \Pi_{T^{i}}(v, y) \\
& =0 .
\end{aligned}
$$

Theorem 9. Let $L \subseteq \Sigma_{0}^{*}$. Let $\left\{n_{x}\right\}_{x \in L}$ be a polynomially bounded sequence in N. Let $S$ and $T$ be coin tossing machines. Assume that the following conditions hold:

1. $|y| \geq|x|$ for all $x \in L$ and all $y \in S(x)$.

2. $S(L) \subseteq L$.

3. $\left\{\Pi_{S}(x, \cdot)\right\}_{x \in L}$ and $\left\{\Pi_{T}(x, \cdot)\right\}_{x \in L}$ are s-indistinguishable.

Then $\left\{\Pi_{S^{n_{x}}}(x, \cdot)\right\}_{x \in L}$ and $\left\{\Pi_{T^{n_{x}}}(x, \cdot)\right\}_{x \in L}$ are s-indistinguishable.

Proof. As in the proof of Theorem 7 we obtain

$$
\delta_{S}\left(\Pi_{S n_{x}}(x, \cdot), \Pi_{T^{n_{x}}}(x, \cdot)\right) \leq n_{x} \delta_{S}\left(\Pi_{M_{x} N O_{x}}(x, \cdot), \Pi_{M_{x} N^{\prime} O_{x}}(x, \cdot)\right),
$$

where $M_{x}=S^{n_{x}-i_{x}-1}, N=S, N^{\prime}=T$ and $O_{x}=T^{i_{x}}$. According to the conditions the families $\left\{\Pi_{N}(x, \cdot)\right\}_{x \in L}$ and $\left\{\Pi_{N^{\prime}}(x, \cdot)\right\}_{x \in L}$ are $s$-indistinguishable.

$$
\begin{aligned}
& \delta_{S}\left(\Pi_{M_{x} N O_{x}}(x, \cdot), \Pi_{M_{x} N^{\prime} O_{x}}(x, \cdot)\right) \\
& =\sum_{y \in \Sigma_{0}^{*}}\left|\Pi_{M_{x} N O_{x}}(x, y)-\Pi_{M_{x} N^{\prime} O_{x}}(x, y)\right| \\
& \leq \sum_{y \in \Sigma_{0}^{*}} \sum_{u \in \Sigma_{0}^{*}} \sum_{v \in \Sigma_{0}^{*}}\left|\Pi_{M_{x}}(x, u)\left\{\Pi_{N}(u, v)-\Pi_{N^{\prime}}(u, v)\right) \Pi_{O_{x}}(v, y)\right| \\
& \leq \sum_{u \in \Sigma_{0}^{*}} \Pi_{M_{x}}(x, u) \sum_{v \in \Sigma_{0}^{*}}\left|\left(\Pi_{N}(u, v)-\Pi_{N^{\prime}}(u, v)\right)\right| \underbrace{\sum_{y \in \Sigma_{0}^{*}} \Pi_{O_{x}}(v, y)}_{\leq 1} \\
& \leq \underbrace{\sum_{u \in \Sigma_{0}^{*}} \Pi_{M_{x}}(x, u)}_{\leq 1} \sup _{\substack{u \in M_{x}(x) \\
\operatorname{sun}_{v \in \Sigma_{0}^{*}}}}\left|\Pi_{N}(u, v)-\Pi_{N^{\prime}}(u, v)\right|
\end{aligned}
$$


Then we have for all $k \in \mathbb{N}$

$$
\begin{aligned}
& \lim _{\substack{x \in L \\
|x| \rightarrow \infty}}|x|^{k} \delta_{S}\left(\Pi_{S^{n_{x}}}(x, \cdot), \Pi_{T^{n_{x}}}(x, \cdot)\right) \\
\leq & \lim _{\substack{x \in L \\
\mid x \in \rightarrow \infty}} n_{x}|x|^{k} \sup _{u \in M_{x}(x)} \delta_{S}\left(\Pi_{N}(u, \cdot), \Pi_{N^{\prime}}(u, \cdot)\right) \\
\leq & \lim _{\substack{x \in L \\
|x| \rightarrow \infty}} n|x|^{k+d} \sup _{u \in M_{x}(x)} \delta_{S}\left(\Pi_{N}(u, \cdot), \Pi_{N^{\prime}}(u, \cdot)\right) \\
& \left(\left\{n_{x}\right\}_{x \in L} \text { is polynomially bounded. Therefore there is } d \in \mathbb{N} \text { with } n_{x} \leq|x|^{d}\right) \\
\leq & \limsup _{\substack{u \in L \\
|u|-\infty}}|u|^{k+d} \delta_{S}\left(\Pi_{N}(u, \cdot), \Pi_{N^{\prime}}(u, \cdot)\right) \\
= & 0 .
\end{aligned}
$$

\section{References}

1. M. Garey and D. S. Johnson. Computers and Intractability, A Guide to the Theory of NP-Completeness. Freeman, 1979.

2. O. Goldreich, S. Micali, and A. Widgerson. Proofs that Yield Nothing But Their Validity or All Languages in NP Have Zero-Knowledge Proof Systems. J. ACM Vol. 38, pp. 691-729, 1991.

3. Harry R. Lewis and Christos H. Papadimitriou. Elements of the theory of Compthtation. Prentice-Hall, 1981.

4. K. R. Reischuk. Einführung in die Komplexitätstheorie. Teubner, 1990. 